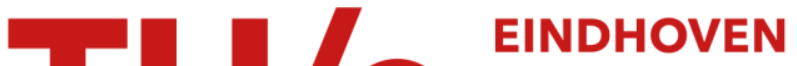 UNIVERSITY OF TECHNOLOGY
}

\section{A modified S-matrix algorithm for the aperiodic Fourier modal method in contrast-field formulation}

\section{Citation for published version (APA):}

Pisarenco, M., Maubach, J. M. L., Setija, I. D., \& Mattheij, R. M. M. (2011). A modified S-matrix algorithm for the aperiodic Fourier modal method in contrast-field formulation. (CASA-report; Vol. 1139). Technische Universiteit Eindhoven.

Document status and date:

Published: 01/01/2011

\section{Document Version:}

Publisher's PDF, also known as Version of Record (includes final page, issue and volume numbers)

\section{Please check the document version of this publication:}

- A submitted manuscript is the version of the article upon submission and before peer-review. There can be important differences between the submitted version and the official published version of record. People interested in the research are advised to contact the author for the final version of the publication, or visit the $\mathrm{DOI}$ to the publisher's website.

- The final author version and the galley proof are versions of the publication after peer review.

- The final published version features the final layout of the paper including the volume, issue and page numbers.

Link to publication

\section{General rights}

Copyright and moral rights for the publications made accessible in the public portal are retained by the authors and/or other copyright owners and it is a condition of accessing publications that users recognise and abide by the legal requirements associated with these rights.

- Users may download and print one copy of any publication from the public portal for the purpose of private study or research.

- You may not further distribute the material or use it for any profit-making activity or commercial gain

- You may freely distribute the URL identifying the publication in the public portal.

If the publication is distributed under the terms of Article 25fa of the Dutch Copyright Act, indicated by the "Taverne" license above, please follow below link for the End User Agreement:

www.tue.nl/taverne

Take down policy

If you believe that this document breaches copyright please contact us at:

openaccess@tue.nl

providing details and we will investigate your claim. 


\section{EINDHOVEN UNIVERSITY OF TECHNOLOGY}

Department of Mathematics and Computer Science

\section{CASA-Report II-39}

June 2OII

A modified S-matrix algorithm for the aperiodic Fourier modal method in contrast-field formulation

\section{by}

M. Pisarenco, J.M.L. Maubach, I. Setija, R.M.M. Mattheij

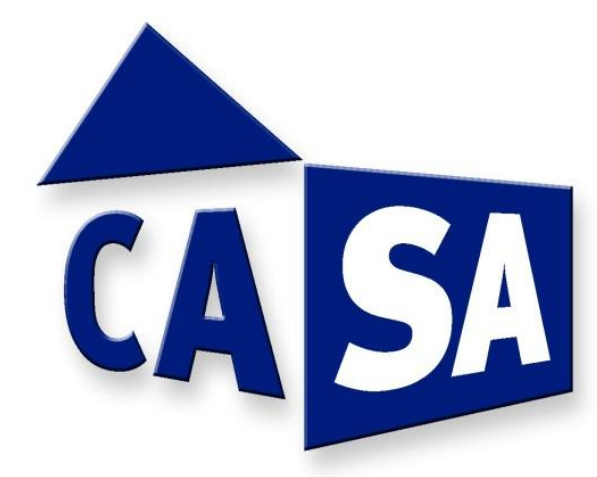

Centre for Analysis, Scientific computing and Applications

Department of Mathematics and Computer Science

Eindhoven University of Technology

P.O. Box 513

5600 MB Eindhoven, The Netherlands

ISSN: 0926-4507 



\title{
A modified S-matrix algorithm for the aperiodic Fourier modal method in contrast-field formulation
}

\author{
Maxim Pisarenco, ${ }^{1, *}$ Joseph Maubach, ${ }^{1}$ Irwan Setija, ${ }^{2}$ Robert Mattheij ${ }^{1}$ \\ ${ }^{1}$ Department of Mathematics and Computer Science, Eindhoven University of Technology, \\ PO Box 513, 5600MB Eindhoven, The Netherlands \\ ${ }^{2}$ Department of Research, ASML Netherlands B. V., \\ De Run 6501, 5504DR Veldhoven, The Netherlands \\ *Corresponding author: m.pisarenco@tue.nl
}

The Fourier modal method (FMM) is a method for efficiently solving Maxwell's equations with periodic boundary conditions. In order to apply the FMM to non-periodic structures, perfectly matched layers need to be placed at the periodic boundaries and the Maxwell equations have to be formulated in terms of a contrast (scattered) field. This reformulation modifies the structure of the resulting linear systems and makes the direct application of available stable recursion algorithms impossible. We adapt the well-known S-matrix algorithm for use with the aperiodic Fourier modal method in contrast field formulation (aFMM-CFF). To this end, stable recursive relations are derived for linear systems with non-homogeneous structure. The stability of the algorithm is confirmed by numerical results.

OCIS codes: 000.4430, 050.1755, 290.2558.

\section{Introduction}

The classical Fourier modal method (FMM) [1,2] and the aperiodic Fourier modal method in contrast-field formulation (aFMM-CFF) [3] are numerical solution methods of the Maxwell equations for respectively infinitely periodic and finite structures. Both methods consist of two main steps: $\{1\}$ discretizing the computational domain into slices and obtaining the general solution (up to integration constants) in each slice and $\{2\}$ determining the integration constants (or modal field amplitudes) by solving a sequence of recursive linear systems resulting from the application of the interface conditions. The straightforward approach for solving the sequence of linear systems, the T-matrix algorithm, is known to be numerically 
unstable [4]. This issue is common for different numerical methods in optics and electromagnetics when discretizing the direction normal to the layered media and is generally linked to the growing exponentials appearing in the equations.

During the past two decades many algorithms for solving the sequence of recursive linear systems which avoid the instability issues have been proposed [4-7]. Many of them are, in one way or another, connected to the S-matrix algorithm [8]. Even for the enhancedtransmittance matrix approach [9], which has a somewhat different algebraic structure, the connection to the S-matrix algorithm has been revealed [10]. A recent study [11] shows that the enhanced-transmittance matrix approach is equivalent to a stable condensation algorithm based on Riccati transformations. For a stability study of the latter see [12] and references therein. We refer the reader to Ref. [13] for an account of the S-matrix, and related R-matrix algorithms. It is worth mentioning that an efficient parallel implementation of the S-matrix algorithm has been recently presented [14].

The S-matrix algorithm relies on the physical concept of mapping the incoming waves on an interface to outgoing waves. This mapping is realized by a so-called S-matrix. Therefore the S-matrix algorithm is suited for linear systems with a homogeneous structure (of the type $\mathbf{A}_{l} \mathbf{x}_{l}=\mathbf{A}_{l+1} \mathbf{x}_{l+1}$, as opposed to the more general non-homogeneous case $\mathbf{A}_{l} \mathbf{x}_{l}+\mathbf{f}_{l}=$ $\left.\mathbf{A}_{l+1} \mathbf{x}_{l+1}+\mathbf{f}_{l+1}\right)$. In the aFMM-CFF, the modification of the interface conditions and of the general solution determines a non-homogeneous structure of the linear systems. We adapt the existing S-matrix algorithm to the new structure of the equations. The choice of the S-matrix approach over the enhanced transmittance matrix approach as a starting point for the extension is explained by the superior flexibility and generality of the former.

This paper is structured as follows. In Section 2 we generalize the presentation in [3] to arbitrary shapes and derive the recursive linear systems for the three fundamental cases (TE, TM, conical). In Section 3 we demonstrate how the classical S-matrix algorithm can be adapted to the new structure of the recursive linear systems. Section 4 contains numerical results confirming the stability of the proposed method. The problem of scattering from a dielectric cylinder, which admits a semi-analytical solution, is used for this purpose. Finally, our conclusions are presented in the last section.

\section{The contrast-field formulation and the structure of the recursive linear sys- tems}

In this section we show the consequences of the contrast-field formulation on the structure of the recursive linear systems. In [3] the aFMM-CFF is presented in detail for the case of rectangular shapes and planar incidence with TE-polarization. In order to clearly define the recursive linear systems, we present here in a brief manner all the three cases (TE, TM, conical) for arbitrary shapes (arbitrary number of slices). 


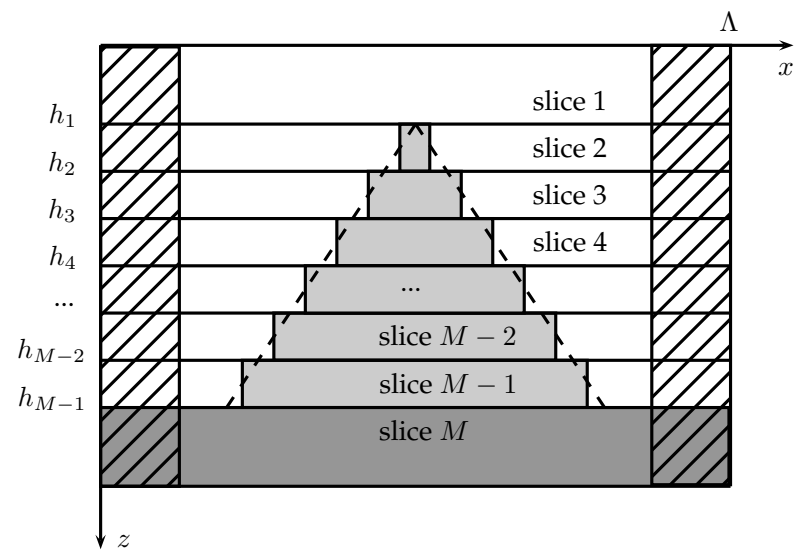

Fig. 1. Sliced geometry. The dashed line represents the smooth profile being approximated. The hatched areas indicate PMLs.

We assume an incident field $\mathbf{e}^{i n c}=\mathbf{a} e^{-i \mathbf{k}^{i n c} \cdot \mathbf{x}}$ and a domain $(x, y, z)=[0, \Lambda] \times \mathbb{R} \times \mathbb{R}$. The equations are discretized in the $z$-direction by subdivision into (thin) slices. In a slice $l$ the permittivity $\epsilon_{l}(x)$ is considered $z$-independent. This procedure replaces a smooth profile by a staircase, as shown in Fig. 1. The Maxwell equations for the contrast field constitute the backbone for further presentation. They are derived from the Maxwell equations for the total field,

$$
\nabla \times \mathbf{e}_{l}^{t}(\mathbf{x})=-k_{0} \mathbf{h}_{l}^{t}(\mathbf{x}), \quad \nabla \times \mathbf{h}_{l}^{t}(\mathbf{x})=-k_{0} \epsilon_{l}(x) \mathbf{e}_{l}^{t}(\mathbf{x})
$$

and the Maxwell equations for the corresponding background field,

$$
\nabla \times \mathbf{e}_{l}^{b}(\mathbf{x})=-k_{0} \mathbf{h}_{l}^{b}(\mathbf{x}), \quad \nabla \times \mathbf{h}_{l}^{b}(\mathbf{x})=-k_{0} \epsilon_{l}^{b} \mathbf{e}_{l}^{b}(\mathbf{x}) .
$$

The geometry corresponding to $\epsilon_{l}$ consists of a background multilayer on top of which a bounded scatterer is placed. The background permittivity $\epsilon_{l}^{b}$ is given by the permittivity of the background multilayer. For the geometry presented in Fig. $1, \epsilon_{l}^{b}=\epsilon_{1}$ for $l=1, \ldots, M-1$ and $\epsilon_{M}^{b}=\epsilon_{M}$. Note that the background problem admits an analytical solution. Lateral radiation conditions for the contrast field are imposed with the help of perfectly matched layers (PMLs) [15], implemented by a complex coordinate transformation [16]. For PMLs placed in the $x$-directions, this implies a change of the $x$-derivative in the differential equations (1), (2) as follows

$$
\frac{\partial}{\partial x} \rightarrow \frac{1}{f^{\prime}(x)} \frac{\partial}{\partial x},
$$


where the coordinate transformation $f(x)$ has a polynomial variation of the imaginary part in the PMLs

$$
f(x)= \begin{cases}x+i \sigma_{0}\left|x-x_{l}\right|^{(p+1)} /(p+1), & 0 \leq x \leq x_{l}, \\ x, & x_{l}<x<x_{r} \\ x-i \sigma_{0}\left|x-x_{r}\right|^{(p+1)} /(p+1), & x_{r} \leq x \leq \Lambda,\end{cases}
$$

The PMLs are placed in the regions $x \in\left[0, x_{l}\right]$ and $x \in\left[x_{r}, \Lambda_{x}\right]$. To discretize in the $x$ direction we use a Galerkin approach with Fourier harmonics as basis functions and test functions,

$$
\phi_{n}(x, y)=e^{-i\left(k_{x n} x+k_{y} y\right)}
$$

where

$$
k_{x n}=k_{x}^{i n c}+n \frac{2 \pi}{\Lambda}, \quad k_{y}=k_{y}^{i n c}, \quad \text { for } n=-N \ldots+N .
$$

In each slice $l$ the total and background fields are expanded as

$$
\begin{aligned}
& e_{\alpha, l}^{t / b}(x, y, z)=\sum_{n=-N}^{N} s_{\alpha, l, n}^{t / b}(z) \phi_{n}(x, y)=\left(\mathbf{s}_{\alpha, l}^{t / b}(z)\right)^{T} \cdot \phi(x, y), \\
& h_{\alpha, l}^{t / b}(x, y, z)=\sum_{n=-N}^{N} u_{\alpha, l, n}^{t / b}(z) \phi_{n}(x, y)=\left(\mathbf{u}_{\alpha, l}^{t / b}(z)\right)^{T} \cdot \phi(x, y) .
\end{aligned}
$$

The $\alpha$ symbol stands for the $x_{-}, y$-, or $z$-component of the field. Although the background field may be represented exactly by only the $\phi_{0}$ function, we use the general formulation in order to get a system for the background field which is formally of the same size as the one for the total field. We apply the Galerkin method with a standard inner product on the interval $[0, \Lambda]$ to the total field equations (1) and background field equations (2) and subsequently take the difference of the discrete sets of equations. Denoting $\mathbf{s}_{\alpha, l}^{c}=\mathbf{s}_{\alpha, l}^{t}-\mathbf{s}_{\alpha, l}^{b}$ and $\mathbf{u}_{\alpha, l}^{c}=\mathbf{u}_{\alpha, l}^{t}-\mathbf{u}_{\alpha, l}^{b}$, we obtain the discretized equations for the contrast field,

$$
\begin{aligned}
-i \mathbf{K}_{y} \mathbf{s}_{z, l}^{c}(z)-k_{0}^{-1} \frac{d}{d z} \mathbf{s}_{y, l}^{c}(z) & =-\mathbf{u}_{x, l}^{c}(z), \\
k_{0}^{-1} \frac{d}{d z} \mathbf{s}_{x, l}^{c}(z)+i \mathbf{F} \mathbf{K}_{x} \mathbf{s}_{z, l}^{c}(z) & =-\mathbf{u}_{y, l}^{c}(z), \\
-i \mathbf{F} \mathbf{K}_{x} \mathbf{s}_{y, l}^{c}(z)+i \mathbf{K}_{y} \mathbf{s}_{x, l}^{c}(z) & =-\mathbf{u}_{z, l}^{c}(z), \\
-i \mathbf{K}_{y} \mathbf{u}_{z, l}^{c}(z)-k_{0}^{-1} \frac{d}{d z} \mathbf{u}_{y, l}^{c}(z) & =-\mathbf{P}_{l}^{-1} \mathbf{s}_{x, l}^{c}(z)-\left(\mathbf{P}_{l}^{-1}-\mathbf{P}_{l}^{b-1}\right) \mathbf{s}_{x, l}^{b}(z), \\
k_{0}^{-1} \frac{d}{d z} \mathbf{u}_{x, l}^{c}(z)+i \mathbf{F} \mathbf{K}_{x} \mathbf{u}_{z, l}^{c}(z) & =-\mathbf{E}_{l} \mathbf{s}_{y, l}^{c}(z)-\left(\mathbf{E}_{l}-\mathbf{E}_{l}^{b}\right) \mathbf{s}_{y, l}^{b}(z), \\
-i \mathbf{F} \mathbf{K}_{x} \mathbf{u}_{y, l}^{c}(z)+i \mathbf{K}_{y} \mathbf{u}_{x, l}^{c}(z) & =-\mathbf{E}_{l} \mathbf{s}_{z, l}^{c}(z)-\left(\mathbf{E}_{l}-\mathbf{E}_{l}^{b}\right) \mathbf{s}_{z, l}^{b}(z) .
\end{aligned}
$$


The matrices in the expressions above are defined as follows,

$$
\begin{aligned}
\left(\mathbf{K}_{x}\right)_{m n} & =\left(k_{x n} / k_{0}\right) \delta_{m n} \\
\left(\mathbf{K}_{y}\right)_{m n} & =\left(k_{y} / k_{0}\right) \delta_{m n} \\
\left(\mathbf{E}_{l}\right)_{m n} & =\hat{\epsilon}_{l, n-m} \\
\left(\mathbf{P}_{l}\right)_{m n} & =\hat{p}_{l, n-m} \\
\left(\mathbf{E}_{l}^{b}\right)_{m n} & =\epsilon_{l}^{b} \delta_{m n} \\
\left(\mathbf{P}_{l}^{b}\right)_{m n} & =\left(\epsilon_{l}^{b}\right)^{-1} \delta_{m n} \\
(\mathbf{F})_{m n} & =\hat{\gamma}_{n-m}
\end{aligned}
$$

for $m, n=-N \ldots+N$. Here $\delta_{m n}$ is the Kronecker delta, $\hat{\epsilon}_{l, k}$ is the $k$-th Fourier coefficient of $\epsilon_{l}(x), \hat{p}_{l, k}$ is the $k$-th Fourier coefficient of $p_{l}(x)=1 / \epsilon_{l}(x)$ and $\hat{\gamma}_{k}$ is the $k$-th Fourier coefficient

of $\gamma(x)=\left(\frac{d f}{d x}\right)^{-1}$. The function $f(x)$ is the complex coordinate transformation implementing the PML and is defined in [3]. The Li rules [17] have been applied in (6d). The background field is determined in advance by solving the Fresnel reflection-transmission problem for a multilayer.

$$
\begin{aligned}
& \mathbf{s}_{\alpha, l}^{b}(z)=\mathbf{d}_{0} s_{\alpha, l}^{b}(z)=\mathbf{d}_{0}\left(a_{s, \alpha, l} e^{-k_{0} q_{l}\left(z-h_{l}\right)}+r_{s, \alpha, l} e^{k_{0} q_{l}\left(z-h_{l}\right)}\right), \\
& \mathbf{u}_{\alpha, l}^{b}(z)=\mathbf{d}_{0} u_{\alpha, l}^{b}(z)=\mathbf{d}_{0}\left(a_{u, \alpha, l} e^{-k_{0} q_{l}\left(z-h_{l}\right)}+r_{u, \alpha, l} e^{k_{0} q_{l}\left(z-h_{l}\right)}\right),
\end{aligned}
$$

where $\mathbf{d}_{0} \in \mathbb{R}^{2 N+1}$ is an all-zero vector except for entry $N+1$ and $q_{l}$ is defined as

$$
q_{l}=i \sqrt{\epsilon_{l}^{b}-\left(\frac{k_{x}^{i n c}}{k_{0}}\right)^{2}-\left(\frac{k_{y}^{i n c}}{k_{0}}\right)^{2}}
$$

The coefficients $a_{s, \alpha, l}, r_{s, \alpha, l}$, and $a_{u, \alpha, l}, r_{u, \alpha, l}$ in (8) are the amplitudes of the downward and upward traveling waves corresponding to the electric and magnetic background field.

\section{A. TE-polarization}

In the case of planar incidence and TE-polarization $\left(\mathbf{s}_{x}^{c}=\mathbf{s}_{z}^{c}=\mathbf{u}_{y}^{c}=0\right)$, the Maxwell equations for the contrast field (6) reduce to

$$
\frac{d^{2}}{d z^{2}} \mathbf{s}_{y, l}^{c}(z)=k_{0}^{2} \mathbf{A}_{l} \mathbf{s}_{y, l}^{c}(z)-k_{0}^{2}\left(\mathbf{E}_{l}-\mathbf{E}_{l}^{b}\right) \mathbf{s}_{y, l}^{b}(z)
$$

where $\mathbf{A}_{l}=\left(\mathbf{F K}_{x}\right)^{2}-\mathbf{E}_{l}$. Equation (10) is a system of non-homogeneous second order ordinary differential equations. Its solution is of the form

$$
\mathbf{s}_{y, l}^{c}=\mathbf{s}_{y, h o m, l}^{c}+\mathbf{s}_{y, p a r t, l}^{c}
$$


The homogeneous solution is given by

$$
\mathbf{s}_{y, h o m, l}^{c}(z)=\mathbf{W}_{l}\left(e^{-k_{0} \mathbf{Q}_{l}\left(z-h_{l}\right)} \mathbf{c}_{l}^{+}+e^{k_{0} \mathbf{Q}_{l}\left(z-h_{l+1}\right)} \mathbf{c}_{l}^{-}\right),
$$

where $\mathbf{W}_{l}$ is the matrix of eigenvectors of $\mathbf{A}_{l}$, and $\mathbf{Q}_{l}$ is a diagonal matrix with square roots of the corresponding eigenvalues on its diagonal. We assume the following form for the particular solution (method of undetermined coefficients for systems, see [3])

$$
\mathbf{s}_{y, p a r t, l}^{c}(z)=\mathbf{p}_{l} s_{y, l}^{b}(z),
$$

where $\mathbf{p}_{l} \in \mathbb{R}^{2 N+1}$ is a vector to be determined. Substitution of (13) in (10) yields

$$
\left(\mathbf{A}_{l}-q_{l}^{2} \mathbf{I}\right) \mathbf{p}_{l}=\left(\mathbf{E}_{l}-\mathbf{E}_{l}^{b}\right) \mathbf{d}_{0} .
$$

At the interface, continuity of the tangential components of the fields is required,

$$
\begin{aligned}
\mathbf{s}_{y, l}^{c}\left(h_{l+1}\right) & =\mathbf{s}_{y, l+1}^{c}\left(h_{l+1}\right), \\
\mathbf{u}_{x, l}^{c}\left(h_{l+1}\right) & =\mathbf{u}_{x, l+1}^{c}\left(h_{l+1}\right) .
\end{aligned}
$$

These conditions hold for the contrast field as a result of the continuity of tangential components of the total and background fields. Using (6a) yields

$$
\begin{aligned}
\mathbf{s}_{y, l}^{c}\left(h_{l+1}\right) & =\mathbf{s}_{y, l+1}^{c}\left(h_{l+1}\right), \\
k_{0}^{-1} \frac{d}{d z} \mathbf{s}_{y, l}^{c}\left(h_{l+1}\right) & =k_{0}^{-1} \frac{d}{d z} \mathbf{s}_{y, l+1}^{c}\left(h_{l+1}\right) .
\end{aligned}
$$

We define $\mathbf{X}_{l}=e^{-k_{0} \mathbf{Q}_{l}\left(h_{l+1}-h_{l}\right)}$ and $\mathbf{V}_{l}=-\mathbf{W}_{l} \mathbf{Q}_{l}$. Then, from (16), (11), (12), (13) we have for each slice

$$
\left[\begin{array}{cc}
\mathbf{W}_{l} \mathbf{X}_{l} & \mathbf{W}_{l} \\
\mathbf{V}_{l} \mathbf{X}_{l} & -\mathbf{V}_{l}
\end{array}\right]\left[\begin{array}{c}
\mathbf{c}_{l}^{+} \\
\mathbf{c}_{l}^{-}
\end{array}\right]+\mathbf{g}_{l}\left(h_{l+1}\right)=\left[\begin{array}{cc}
\mathbf{W}_{l+1} & \mathbf{W}_{l+1} \mathbf{X}_{l+1} \\
\mathbf{V}_{l+1} & -\mathbf{V}_{l+1} \mathbf{X}_{l+1}
\end{array}\right]\left[\begin{array}{c}
\mathbf{c}_{l+1}^{+} \\
\mathbf{c}_{l+1}^{-}
\end{array}\right]+\mathbf{g}_{l+1}\left(h_{l+1}\right),
$$

where

$$
\mathbf{g}_{l}(z)=\left[\begin{array}{l}
\mathbf{p}_{l} s_{y, l}^{b} \\
\mathbf{p}_{l} u_{x, l}^{b}
\end{array}\right]
$$

\section{B. TM-polarization}

In the case of planar incidence and TM-polarization $\left(\mathbf{u}_{x}^{c}=\mathbf{u}_{z}^{c}=\mathbf{s}_{y}^{c}=0\right)$, the Maxwell equations for the contrast field (6) reduce to

$$
\frac{d^{2}}{d z^{2}} \mathbf{u}_{y, l}^{c}=\mathbf{P}_{l}^{-1} \mathbf{B}_{l} \mathbf{u}_{y, l}^{c}+\left(\mathbf{P}_{l}^{-1} \mathbf{B}_{l}-\left(\mathbf{P}_{l}^{b}\right)^{-1} \mathbf{B}_{l}^{b}\right) \mathbf{u}_{y, l}^{b},
$$


where $\mathbf{B}_{l}=\left(\mathbf{F} \mathbf{K}_{x} \mathbf{E}_{l}^{-1} \mathbf{F} \mathbf{K}_{x}-\mathbf{I}\right)$ and $\mathbf{B}_{l}^{b}=\left(\mathbf{F} \mathbf{K}_{x}\left(\mathbf{E}_{l}^{b}\right)^{-1} \mathbf{F} \mathbf{K}_{x}-\mathbf{I}\right)$. Equation (19) is obtained by substituting (6d) and (6f) in (6b) and subsequently using the Maxwell equations for the background field to replace $\mathbf{s}_{x, l}^{b}$ and $\mathbf{s}_{z, l}^{b}$ by $\mathbf{u}_{y, l}^{b}$. The solution of (19) is of the form

$$
\mathbf{u}_{y, l}^{c}=\mathbf{u}_{y, h o m, l}^{c}+\mathbf{u}_{y, p a r t, l}^{c}
$$

The homogeneous solution is given by

$$
\mathbf{u}_{y, h o m, l}^{c}(z)=\mathbf{W}_{l}\left(e^{-k_{0} \mathbf{Q}_{l}\left(z-h_{l}\right)} \mathbf{c}_{l}^{+}+e^{k_{0} \mathbf{Q}_{l}\left(z-h_{l+1}\right)} \mathbf{c}_{l}^{-}\right)
$$

where, as before, $\mathbf{W}_{l}$ and $\mathbf{Q}_{l}$ are respectively the matrix of eigenvectors and diagonal matrix of square roots of eigenvalues of $\mathbf{P}_{l}^{-1} \mathbf{B}_{l}$. We assume the following form for the particular solution

$$
\mathbf{u}_{y, p a r t, l}^{c}(z)=\mathbf{p}_{l} u_{y, l}^{b}(z)
$$

Substitution of (22) in (19) yields

$$
\left(\mathbf{P}_{l}^{-1} \mathbf{B}_{l}-q_{l}^{2} \mathbf{I}\right) \mathbf{p}_{l}=\left(\left(\mathbf{P}_{l}^{b}\right)^{-1} \mathbf{B}_{l}^{b}-\mathbf{P}_{l}^{-1} \mathbf{B}_{l}\right) \mathbf{d}_{0}
$$

At the interface, continuity of the tangential components of the fields is required,

$$
\begin{aligned}
& \mathbf{u}_{y, l}^{c}\left(h_{l+1}\right)=\mathbf{u}_{y, l+1}^{c}\left(h_{l+1}\right), \\
& \mathbf{s}_{x, l}^{c}\left(h_{l+1}\right)=\mathbf{s}_{x, l+1}^{c}\left(h_{l+1}\right) .
\end{aligned}
$$

Using (6d) yields

$$
\begin{gathered}
\mathbf{u}_{y, l}^{c}\left(h_{l+1}\right)=\mathbf{u}_{y, l+1}^{c}\left(h_{l+1}\right), \\
k_{0}^{-1} \mathbf{P}_{l} \frac{d}{d z} \mathbf{u}_{y, l}^{c}\left(h_{l+1}\right)-\mathbf{P}_{l}\left(\mathbf{P}_{l}^{-1}-\left(\mathbf{P}_{l}^{b}\right)^{-1}\right) \mathbf{s}^{b}{ }_{x, l}\left(h_{l+1}\right)= \\
k_{0}^{-1} \mathbf{P}_{l+1} \frac{d}{d z} \mathbf{u}_{y, l+1}^{c}\left(h_{l+1}\right)-\mathbf{P}_{l+1}\left(\mathbf{P}_{l+1}^{-1}-\left(\mathbf{P}_{l+1}^{b}\right)^{-1}\right) \mathbf{s}^{b}{ }_{x, l+1}\left(h_{l+1}\right) .
\end{gathered}
$$

We define $\mathbf{V}_{l}=-\mathbf{P}_{l} \mathbf{W}_{l} \mathbf{Q}_{l}$ (keeping the old definition of $\mathbf{X}_{l}$ ). Then, from (25), (20), (21), (22) we have for each slice

$$
\left[\begin{array}{cc}
\mathbf{W}_{l} \mathbf{X}_{l} & \mathbf{W}_{l} \\
\mathbf{V}_{l} \mathbf{X}_{l} & -\mathbf{V}_{l}
\end{array}\right]\left[\begin{array}{l}
\mathbf{c}_{l}^{+} \\
\mathbf{c}_{l}^{-}
\end{array}\right]+\mathbf{g}_{l}\left(h_{l+1}\right)=\left[\begin{array}{cc}
\mathbf{W}_{l+1} & \mathbf{W}_{l+1} \mathbf{X}_{l+1} \\
\mathbf{V}_{l+1} & -\mathbf{V}_{l+1} \mathbf{X}_{l+1}
\end{array}\right]\left[\begin{array}{l}
\mathbf{c}_{l+1}^{+} \\
\mathbf{c}_{l+1}^{-}
\end{array}\right]+\mathbf{g}_{l+1}\left(h_{l+1}\right)
$$

where

$$
\mathbf{g}_{l}(z)=\left[\begin{array}{c}
\mathbf{p}_{l} u_{y, l}^{b} \\
\left(\mathbf{P}_{l} \mathbf{p}_{l}+\left(\mathbf{P}_{l}-\epsilon_{l}^{b} \mathbf{I}\right) \mathbf{d}_{0}\right) s_{x, l}^{b}
\end{array}\right]
$$




\section{C. Conical incidence}

In the case of conical incidence, the Maxwell equations for the contrast field (6) reduce to

$$
\begin{aligned}
& \frac{d^{2}}{d z^{2}} \mathbf{s}_{x, l}^{c}(z)=k_{0}^{2} \mathbf{C}_{l} \mathbf{s}_{x, l}^{c}(z)+k_{0}^{2}\left(\mathbf{B}_{l} \mathbf{P}_{l}^{-1}-\mathbf{B}_{l}^{b}\left(\mathbf{P}_{l}^{b}\right)^{-1}\right) \mathbf{s}_{x, l}^{b}(z), \\
& \frac{d^{2}}{d z^{2}} \mathbf{u}_{x, l}^{c}(z)=k_{0}^{2} \mathbf{D}_{l} \mathbf{u}_{x, l}^{c}(z)-k_{0}^{2}\left(\mathbf{E}_{l}-\mathbf{E}_{l}^{b}\right) \mathbf{u}_{x, l}^{b}(z),
\end{aligned}
$$

where $\mathbf{C}_{l}=\mathbf{K}_{y}^{2}+\mathbf{B}_{l} \mathbf{P}_{l}^{-1}, \mathbf{D}_{l}=\mathbf{K}_{y}^{2}+\mathbf{A}_{l}$. As for the TM-polarization, in order to arrive at (28) from (6), additionally the Maxwell equations for the background field need to be used. The solution vector is of the form

$$
\begin{gathered}
\mathbf{s}_{x, l}^{c}=\mathbf{s}_{x, h o m, l}^{c}+\mathbf{s}_{x, \text { part }, l}^{c} \\
\mathbf{u}_{x, l}^{c}=\mathbf{u}_{x, h o m, l}^{c}+\mathbf{u}_{x, \text { part }, l}^{c} .
\end{gathered}
$$

The homogeneous solution is given by

$$
\begin{aligned}
\mathbf{s}_{x, h o m, l}^{c}(z) & =\mathbf{W}_{s, l}\left(e^{-k_{0} \mathbf{Q}_{s, l}\left(z-h_{l}\right)} \mathbf{c}_{s, l}^{+}+e^{k_{0} \mathbf{Q}_{s, l}\left(z-h_{l+1}\right)} \mathbf{c}_{s, l}^{-}\right) \\
\mathbf{u}_{x, h o m, l}^{c}(z) & =\mathbf{W}_{u, l}\left(e^{-k_{0} \mathbf{Q}_{u, l}\left(z-h_{l}\right)} \mathbf{c}_{u, l}^{+}+e^{k_{0} \mathbf{Q}_{u, l}\left(z-h_{l+1}\right)} \mathbf{c}_{u, l}^{-}\right)
\end{aligned}
$$

where the pairs $\mathbf{W}_{s, l}, \mathbf{Q}_{s, l}$ and $\mathbf{W}_{u, l}, \mathbf{Q}_{u, l}$ contain the matrix of eigenvectors and the diagonal matrix of square roots of eigenvalues of, respectively, $\mathbf{C}_{l}$ and $\mathbf{D}_{l}$. To find the particular solution we assume the form

$$
\begin{aligned}
\mathbf{s}_{x, \text { art }, l}^{c}(z) & =\mathbf{p}_{s, l} s_{x, l}^{b}(z), \\
\mathbf{u}_{x, \text { art }, l}^{c}(z) & =\mathbf{p}_{u, l} u_{x, l}^{b}(z) .
\end{aligned}
$$

Using this ansatz in Equation (28), we obtain two linear systems which can be solved for $\mathbf{p}_{s, l}$ and $\mathbf{p}_{u, l}$

$$
\begin{aligned}
\left(\mathbf{C}_{l}-q_{l}^{2} \mathbf{I}\right) \mathbf{p}_{s, l} & =-\left(\mathbf{B}_{l} \mathbf{P}_{l}^{-1}-\mathbf{B}_{l}^{b}\left(\mathbf{P}_{l}^{b}\right)^{-1}\right) \mathbf{d}_{0} \\
\left(\mathbf{D}_{l}-q_{l}^{2} \mathbf{I}\right) \mathbf{p}_{u, l} & =\left(\mathbf{E}_{l}-\mathbf{E}_{l}^{b}\right) \mathbf{d}_{0}
\end{aligned}
$$

At the interface, continuity of the tangential components of the fields is required

$$
\begin{aligned}
\mathbf{s}_{x, l}^{c}\left(h_{l+1}\right) & =\mathbf{s}_{x, l+1}^{c}\left(h_{l+1}\right), \\
\mathbf{s}_{y, l}^{c}\left(h_{l+1}\right) & =\mathbf{s}_{y, l+1}^{c}\left(h_{l+1}\right), \\
\mathbf{u}_{x, l}^{c}\left(h_{l+1}\right) & =\mathbf{u}_{x, l+1}^{c}\left(h_{l+1}\right), \\
\mathbf{u}_{y, l}^{c}\left(h_{l+1}\right) & =\mathbf{u}_{y, l+1}^{c}\left(h_{l+1}\right) .
\end{aligned}
$$


Using (6c) in (6e) and (6f) in (6b), the $y$-components of the fields are expressed in terms of $x$-components,

$$
\begin{aligned}
& \mathbf{s}_{y, l}^{c}=\mathbf{A}_{l}^{-1}\left(\mathbf{F} \mathbf{K}_{x} \mathbf{K}_{y} \mathbf{s}_{x, l}^{c}+k_{0}^{-1} \frac{d}{d z} \mathbf{u}_{x, l}^{c}+\left(\mathbf{E}_{l}-\mathbf{E}_{l}^{b}\right) \mathbf{s}_{y, l}^{b}\right), \\
& \mathbf{u}_{y, l}^{c}=\mathbf{B}_{l}^{-1}\left(\mathbf{F} \mathbf{K}_{x} \mathbf{E}^{-1} \mathbf{K}_{y} \mathbf{u}_{x, l}^{c}+k_{0}^{-1} \frac{d}{d z} \mathbf{s}_{x, l}^{c}-i \mathbf{F} \mathbf{K}_{x}\left(\mathbf{I}-\mathbf{E}_{l}^{b} \mathbf{E}_{l}^{-1}\right) \mathbf{s}_{z, l}^{b}\right) .
\end{aligned}
$$

We define

$$
\begin{aligned}
\mathbf{W}_{l} & =\left[\begin{array}{cc}
\mathbf{0} & \mathbf{W}_{s, l} \\
\mathbf{A}_{l}^{-1} \mathbf{W}_{u, l} \mathbf{Q}_{u, l} & \mathbf{A}_{l}^{-1} \mathbf{F} \mathbf{K}_{x} \mathbf{K}_{y} \mathbf{W}_{s, l}
\end{array}\right] \\
\mathbf{V}_{l} & =-\left[\begin{array}{cc}
\mathbf{W}_{u, l} & \mathbf{0} \\
\mathbf{B}_{l}^{-1} \mathbf{F} \mathbf{K}_{x} \mathbf{E}_{l}^{-1} \mathbf{K}_{y} \mathbf{W}_{u, l} & \mathbf{B}_{l}^{-1} \mathbf{W}_{s, l} \mathbf{Q}_{s, l}
\end{array}\right]
\end{aligned}
$$

and

$$
\mathbf{c}_{l}^{+}=\left[\begin{array}{c}
-\mathbf{c}_{u, l}^{+} \\
\mathbf{c}_{s, l}^{+}
\end{array}\right], \quad \mathbf{c}_{l}^{-}=\left[\begin{array}{c}
\mathbf{c}_{u, l}^{-} \\
\mathbf{c}_{s, l}^{-}
\end{array}\right] .
$$

Then, from (34), (29), (30), (32) we have for each slice

$$
\left[\begin{array}{cc}
\mathbf{W}_{l} \mathbf{X}_{l} & \mathbf{W}_{l} \\
\mathbf{V}_{l} \mathbf{X}_{l} & -\mathbf{V}_{l}
\end{array}\right]\left[\begin{array}{c}
\mathbf{c}_{l}^{+} \\
\mathbf{c}_{l}^{-}
\end{array}\right]+\mathbf{g}_{l}\left(h_{l+1}\right)=\left[\begin{array}{cc}
\mathbf{W}_{l+1} & \mathbf{W}_{l+1} \mathbf{X}_{l+1} \\
\mathbf{V}_{l+1} & -\mathbf{V}_{l+1} \mathbf{X}_{l+1}
\end{array}\right]\left[\begin{array}{c}
\mathbf{c}_{l+1}^{+} \\
\mathbf{c}_{l+1}^{-}
\end{array}\right]+\mathbf{g}_{l+1}\left(h_{l+1}\right),
$$

where

$$
\mathbf{g}_{l}(z)=\left[\begin{array}{c}
\mathbf{p}_{s, l} s_{x, l}^{b} \\
\mathbf{A}_{l}^{-1}\left(\mathbf{F} \mathbf{K}_{x} \mathbf{K}_{y} \mathbf{p}_{s, l} s_{x, l}^{b}+k_{0}^{-1} \mathbf{p}_{u, l} \frac{d}{d z} u_{x, l}^{b}+\left(\mathbf{E}_{l}-\epsilon_{l}^{b} \mathbf{I}\right) \mathbf{d}_{0} s_{y, l}^{b}\right) \\
\mathbf{p}_{u, l} u_{x, l}^{b} \\
\mathbf{B}_{l}^{-1}\left(\mathbf{F} \mathbf{K}_{x} \mathbf{E}^{-1} \mathbf{K}_{y} \mathbf{p}_{u, l} u_{x, l}^{b}+k_{0}^{-1} \mathbf{p}_{s, l} \frac{d}{d z} s_{x, l}^{b}-i \mathbf{F} \mathbf{K}_{x}\left(\mathbf{I}-\epsilon_{l}^{b} \mathbf{E}_{l}^{-1}\right) \mathbf{d}_{0} s_{z, l}^{b}\right)
\end{array}\right]
$$

with $s_{z, l}^{b}=\frac{i}{\epsilon^{b}}\left(k_{x 0} u_{y, l}^{b}-k_{y} u_{x, l}^{b}\right)$.

\section{Modified S-matrix algorithm}

We now proceed to adapt the standard S-matrix algorithm for use with non-homogeneous recursive linear systems. As shown in Section 2, matching of the interface conditions for the contrast field at interface $l$ yields an equation of the form

$$
\mathbf{R}_{l}\left[\begin{array}{c}
\mathbf{X}_{l} \mathbf{c}_{l}^{+} \\
\mathbf{c}_{l}^{-}
\end{array}\right]+\mathbf{g}_{l}\left(h_{l+1}\right)=\mathbf{R}_{l+1}\left[\begin{array}{c}
\mathbf{c}_{l+1}^{+} \\
\mathbf{X}_{l+1} \mathbf{c}_{l+1}^{-}
\end{array}\right]+\mathbf{g}_{l+1}\left(h_{l+1}\right) .
$$

This is rewritten in the T-matrix formalism as

$$
\left[\begin{array}{c}
\mathbf{c}_{l+1}^{+} \\
\mathbf{X}_{l+1} \mathbf{c}_{l+1}^{-}
\end{array}\right]=\left[\begin{array}{cc}
\mathbf{T}_{l}^{11} & \mathbf{T}_{l}^{12} \\
\mathbf{T}_{l}^{21} & \mathbf{T}_{l}^{22}
\end{array}\right]\left[\begin{array}{c}
\mathbf{X}_{l} \mathbf{c}_{l}^{+} \\
\mathbf{c}_{l}^{-}
\end{array}\right]+\left[\begin{array}{c}
\mathbf{g}_{l}^{\prime 1} \\
\mathbf{g}_{l}^{\prime 2}
\end{array}\right]
$$




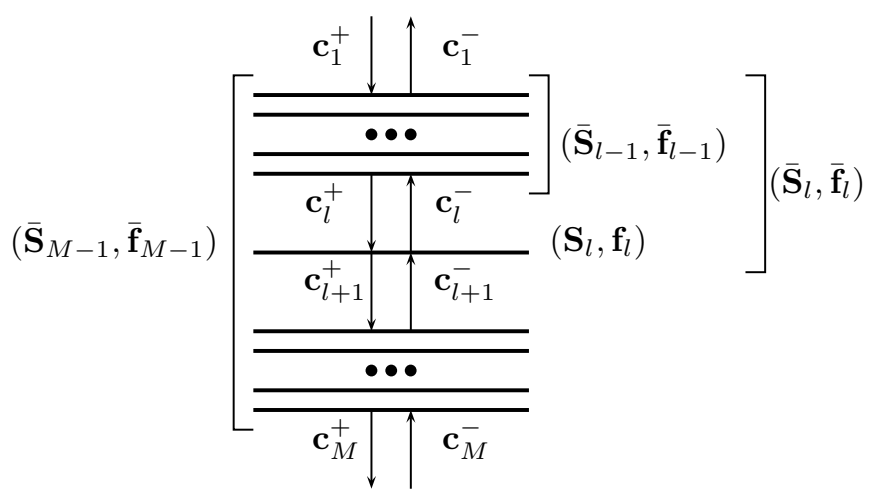

Fig. 2. A stack of interfaces with upward- and downward-traveling waves inbetween.

where

$$
\begin{aligned}
\mathbf{T}_{l} & =\mathbf{R}_{l+1}^{-1} \mathbf{R}_{l}=\frac{1}{2}\left[\begin{array}{cc}
\mathbf{W}_{l+1}^{-1} & \mathbf{V}_{l+1}^{-1} \\
\mathbf{W}_{l+1}^{-1} & -\mathbf{V}_{l+1}^{-1}
\end{array}\right]\left[\begin{array}{cc}
\mathbf{W}_{l} & \mathbf{W}_{l} \\
\mathbf{V}_{l} & -\mathbf{V}_{l}
\end{array}\right], \\
\mathbf{g}_{l}^{\prime} & =\mathbf{R}_{l+1}^{-1}\left(\mathbf{g}_{l}\left(h_{l+1}\right)-\mathbf{g}_{l+1}\left(h_{l+1}\right)\right) .
\end{aligned}
$$

The S-matrix algorithm is derived from the matrix $\mathbf{T}_{l}$ and vector $\mathbf{g}_{l}^{\prime}$ of the T-matrix equation (41). In the spirit of the S-matrix algorithm, the waves scattered at the interface are expressed in terms of waves incident on the interface [13] (see Figure 2)

$$
\left[\begin{array}{c}
\mathbf{c}_{l+1}^{+} \\
\mathbf{c}_{l}^{-}
\end{array}\right]=\left[\begin{array}{ll}
\mathbf{S}_{l}^{11} & \mathbf{S}_{l}^{12} \\
\mathbf{S}_{l}^{21} & \mathbf{S}_{l}^{22}
\end{array}\right]\left[\begin{array}{c}
\mathbf{c}_{l}^{+} \\
\mathbf{c}_{l+1}^{-}
\end{array}\right]+\left[\begin{array}{c}
\mathbf{f}_{l}^{1} \\
\mathbf{f}_{l}^{2}
\end{array}\right] .
$$

The S-matrix $\mathbf{S}_{l}$ and the vector $\mathbf{f}_{l}$ can be determined from the T-matrix $\mathbf{T}_{l}$ and the vector $\mathbf{g}_{l}^{\prime}$ by bringing (41) to form (43).

$$
\begin{aligned}
\mathbf{S}_{l}^{11} & =\left(\mathbf{T}_{l}^{11}-\mathbf{T}_{l}^{12}\left(\mathbf{T}_{l}^{22}\right)^{-1} \mathbf{T}_{l}^{21}\right) \mathbf{X}_{l} \\
\mathbf{S}_{l}^{12} & =\mathbf{T}_{l}^{12}\left(\mathbf{T}_{l}^{22}\right)^{-1} \mathbf{X}_{l+1} \\
\mathbf{S}_{l}^{21} & =-\left(\mathbf{T}_{l}^{22}\right)^{-1} \mathbf{T}_{l}^{21} \mathbf{X}_{l} \\
\mathbf{S}_{l}^{22} & =\left(\mathbf{T}_{l}^{22}\right)^{-1} \mathbf{X}_{l+1} \\
\mathbf{f}_{l}^{1} & =\mathbf{g}_{l}^{\prime 1}-\mathbf{T}_{l}^{12}\left(\mathbf{T}_{l}^{22}\right)^{-1} \mathbf{g}_{l}^{\prime 2} \\
\mathbf{f}_{l}^{2} & =-\left(\mathbf{T}_{l}^{22}\right)^{-1} \mathbf{g}_{l}^{\prime 2}
\end{aligned}
$$

Expressions (43) and (44) describe the scattering properties of the interface $l$. These properties are defined by a local scattering matrix $\mathbf{S}_{l}$ and a local source vector $\mathbf{f}_{l}$. To simplify further 
presentation we denote them together as $\left(\mathbf{S}_{l}, \mathbf{f}_{l}\right)$. Figure 2 gives a schematic representation of the interfaces and associated scattering matrix-vector pairs.

We proceed by defining a cumulative scattering matrix and a cumulative source vector for a stack of multiple interfaces. The matrix-vector pair $\left(\overline{\mathbf{S}}_{l}, \overline{\mathbf{f}}_{l}\right)$ defines the scattering properties of the stack of interfaces $1, \ldots, l$ (see also Figure 2 ).

$$
\left[\begin{array}{c}
\mathbf{c}_{l+1}^{+} \\
\mathbf{c}_{1}^{-}
\end{array}\right]=\left[\begin{array}{ll}
\overline{\mathbf{S}}_{l}^{11} & \overline{\mathbf{S}}_{l}^{12} \\
\overline{\mathbf{S}}_{l}^{21} & \overline{\mathbf{S}}_{l}^{22}
\end{array}\right]\left[\begin{array}{c}
\mathbf{c}_{1}^{+} \\
\mathbf{c}_{l+1}^{-}
\end{array}\right]+\left[\begin{array}{c}
\overline{\mathbf{f}}_{l}^{1} \\
\overline{\mathbf{f}}_{l}^{2}
\end{array}\right] .
$$

By assuming that the cumulative scattering matrix-vector pair for interface $(l-1)$ is known, we will derive the cumulative matrix-vector pair for interface $l$ using the local scattering matrix-vector of interface $l$, as illustrated by the diagram

$$
\left(\overline{\mathbf{S}}_{l-1}, \overline{\mathbf{f}}_{l-1}\right) \stackrel{\left(\mathbf{S}_{l}, \mathbf{f}_{l}\right)}{\longrightarrow}\left(\overline{\mathbf{S}}_{l}, \overline{\mathbf{f}}_{l}\right) .
$$

This defines a recursive relation for the cumulative matrix-vector. Note that the cumulative scattering matrix-vector and the local scattering matrix-vector for interface one are equal,

$$
\left(\overline{\mathbf{S}}_{1}, \overline{\mathbf{f}}_{1}\right)=\left(\mathbf{S}_{1}, \mathbf{f}_{1}\right)
$$

This relation is used to initialize the recursion.

We now outline the derivation of the recursion formally represented in (46). For convenience, relation (45) is repeated here for interface $l-1$

$$
\left[\begin{array}{l}
\mathbf{c}_{l}^{+} \\
\mathbf{c}_{1}^{-}
\end{array}\right]=\left[\begin{array}{ll}
\overline{\mathbf{S}}_{l-1}^{11} & \overline{\mathbf{S}}_{l-1}^{12} \\
\overline{\mathbf{S}}_{l-1}^{21} & \overline{\mathbf{S}}_{l-1}^{22}
\end{array}\right]\left[\begin{array}{l}
\mathbf{c}_{1}^{+} \\
\mathbf{c}_{l}^{-}
\end{array}\right]+\left[\begin{array}{l}
\overline{\mathbf{f}}_{l-1}^{1} \\
\overline{\mathbf{f}}_{l-1}^{2}
\end{array}\right] .
$$

The first equation of (48) and the second equation of (43) yield

$$
\mathbf{c}_{l}^{+}=\mathbf{H}_{l}^{\prime} \overline{\mathbf{S}}_{l-1}^{11} \mathbf{c}_{1}^{+}+\overline{\mathbf{S}}_{l-1}^{12} \mathbf{H}_{l}^{\prime \prime} \mathbf{S}_{l}^{22} \mathbf{c}_{l+1}^{-}+\mathbf{H}_{l}^{\prime} \overline{\mathbf{S}}_{l-1}^{12} \mathbf{f}_{l}^{2}+\mathbf{H}_{l}^{\prime} \overline{\mathbf{f}}_{l-1}^{1},
$$

where

$$
\begin{aligned}
\mathbf{H}_{l}^{\prime} & =\left(\mathbf{I}-\overline{\mathbf{S}}_{l-1}^{12} \mathbf{S}_{l}^{21}\right)^{-1}, \\
\mathbf{H}_{l}^{\prime \prime} & =\left(\mathbf{I}-\mathbf{S}_{l}^{21} \overline{\mathbf{S}}_{l-1}^{12}\right)^{-1} .
\end{aligned}
$$

From the first equation of (43) and (49)

$$
\begin{aligned}
\mathbf{c}_{l+1}^{+}= & \mathbf{S}_{l}^{11} \mathbf{H}_{l}^{\prime} \overline{\mathbf{S}}_{l-1}^{11} \mathbf{c}_{1}^{+}+\left(\mathbf{S}_{l}^{12}+\mathbf{S}_{l}^{11} \overline{\mathbf{S}}_{l-1}^{12} \mathbf{H}_{l}^{\prime \prime} \mathbf{S}_{l}^{22}\right) \mathbf{c}_{l+1}^{-} \\
& +\mathbf{S}_{l}^{11}\left(\mathbf{H}_{l}^{\prime} \overline{\mathbf{S}}_{l-1}^{12} \mathbf{f}_{l}^{2}+\mathbf{H}_{l}^{\prime} \overline{\mathbf{f}}_{l-1}^{1}\right)+\mathbf{f}_{l}^{1} .
\end{aligned}
$$

From the second equation of (48), the second equation of (43) and (49)

$$
\begin{aligned}
\mathbf{c}_{1}^{-}= & \left(\overline{\mathbf{S}}_{l-1}^{21}+\overline{\mathbf{S}}_{l-1}^{22} \mathbf{S}_{l}^{21} \mathbf{H}_{l}^{\prime} \overline{\mathbf{S}}_{l-1}^{11}\right) \mathbf{c}_{1}^{+}+\overline{\mathbf{S}}_{l-1}^{22} \mathbf{H}_{l}^{\prime \prime} \mathbf{S}_{l}^{22} \mathbf{c}_{l+1}^{-} \\
& +\overline{\mathbf{S}}_{l-1}^{22} \mathbf{S}_{l}^{21}\left(\mathbf{H}_{l}^{\prime} \overline{\mathbf{S}}_{l-1}^{12} \mathbf{f}_{l}^{2}+\mathbf{H}_{l}^{\prime} \overline{\mathbf{f}}_{l-1}^{1}\right)+\overline{\mathbf{S}}_{l-1}^{22} \mathbf{f}_{l}^{2}+\overline{\mathbf{f}}_{l-1}^{2} .
\end{aligned}
$$


Now (52) and (53) give the recursion relations

$$
\begin{aligned}
& {\left[\begin{array}{ll}
\overline{\mathbf{S}}_{l}^{11} & \overline{\mathbf{S}}_{l}^{12} \\
\overline{\mathbf{S}}_{l}^{21} & \overline{\mathbf{S}}_{l}^{22}
\end{array}\right]=\left[\begin{array}{cc}
\mathbf{S}_{l}^{11} \mathbf{H}_{l}^{\prime} \overline{\mathbf{S}}_{l-1}^{11} & \mathbf{S}_{l}^{12}+\mathbf{S}_{l}^{11} \overline{\mathbf{S}}_{l-1}^{12} \mathbf{H}_{l}^{\prime \prime} \mathbf{S}_{l}^{22} \\
\overline{\mathbf{S}}_{l-1}^{21}+\overline{\mathbf{S}}_{l-1}^{22} \mathbf{S}_{l}^{21} \mathbf{H}_{l}^{\prime} \overline{\mathbf{S}}_{l-1}^{11} & \overline{\mathbf{S}}_{l-1}^{22} \mathbf{H}_{l}^{\prime \prime} \mathbf{S}_{l}^{22}
\end{array}\right],} \\
& {\left[\begin{array}{c}
\overline{\mathbf{f}}_{l}^{1} \\
\overline{\mathbf{f}}_{l}^{2}
\end{array}\right]=\left[\begin{array}{c}
\mathbf{S}_{l}^{11}\left(\mathbf{H}_{l}^{\prime} \overline{\mathbf{S}}_{l-1}^{12} \mathbf{f}_{l}^{2}+\mathbf{H}_{l}^{\prime} \overline{\mathbf{f}}_{l-1}^{1}\right)+\mathbf{f}_{l}^{1} \\
\overline{\mathbf{S}}_{l-1}^{22} \mathbf{S}_{l}^{21}\left(\mathbf{H}_{l}^{\prime} \overline{\mathbf{S}}_{l-1}^{12} \mathbf{f}_{l}^{2}+\mathbf{H}_{l}^{\prime} \overline{\mathbf{f}}_{l-1}^{1}\right)+\overline{\mathbf{S}}_{l-1}^{22} \mathbf{f}_{l}^{2}+\overline{\mathbf{f}}_{l-1}^{2}
\end{array}\right] .}
\end{aligned}
$$

While the update relation for the matrix $\overline{\mathbf{S}}_{l}$ is the same as in the classical S-matrix algorithm (See Equation (15a) in Ref. [13]), an additional update relation appears for the vector $\overline{\mathbf{f}}_{l}$. The above formulas are recursively used for $l=2, \ldots, M-1$, using (47) for $l=1$. The reflection and transmission coefficients may be computed when the cumulative matrix-vector for all $M-1$ interfaces is known

$$
\left[\begin{array}{c}
\mathbf{c}_{M}^{+} \\
\mathbf{c}_{1}^{-}
\end{array}\right]=\left[\begin{array}{ll}
\overline{\mathbf{S}}_{M-1}^{11} & \overline{\mathbf{S}}_{M-1}^{12} \\
\overline{\mathbf{S}}_{M-1}^{21} & \overline{\mathbf{S}}_{M-1}^{22}
\end{array}\right]\left[\begin{array}{l}
\mathbf{c}_{1}^{+} \\
\mathbf{c}_{M}^{-}
\end{array}\right]+\left[\begin{array}{l}
\overline{\mathbf{f}}_{M-1}^{1} \\
\overline{\mathbf{f}}_{M-1}^{2}
\end{array}\right] .
$$

In the contrast-field formulation of the FMM, the incoming field has been moved into a source term. Therefore, the coefficients corresponding to incoming fields in the superstrate and substrate vanish, $\mathbf{c}_{1}^{+}=\mathbf{c}_{M}^{-}=0$, and we have

$$
\left[\begin{array}{c}
\mathbf{c}_{M}^{+} \\
\mathbf{c}_{1}^{-}
\end{array}\right]=\left[\begin{array}{l}
\overline{\mathbf{f}}_{M-1}^{1} \\
\overline{\mathbf{f}}_{M-1}^{2}
\end{array}\right] \text {. }
$$

Note that for the computation of the vector $\overline{\mathbf{f}}_{M-1}$ in $(54 \mathrm{~b})$ the blocks $\overline{\mathbf{S}}_{l-1}^{11}$ and $\overline{\mathbf{S}}_{l-1}^{21}$ are not required.

Very often, especially for aperiodic structures, we are also interested in the near field, i.e. the field inside and around the scatterer. For this purpose, also the intermediary coefficients in slices $2, \ldots, M-1$ need to be computed in a stable way. This requires the inverse of $\mathbf{S}_{l}^{11}$ and consequently of $\mathbf{X}_{l}$, which causes instabilities due to growing exponentials. To avoid the direct inversion of $\mathbf{X}_{l}$, we separate the propagation matrices from the scattering matrix. Equation (43) is written as

$$
\left[\begin{array}{c}
\mathbf{c}_{l+1}^{+} \\
\mathbf{c}_{l}^{-}
\end{array}\right]=\left[\begin{array}{cc}
\mathbf{S}_{l}^{\prime 11} & \mathbf{S}_{l}^{\prime 12} \\
\mathbf{S}_{l}^{\prime 21} & \mathbf{S}_{l}^{\prime 2}
\end{array}\right]\left[\begin{array}{c}
\mathbf{X}_{l} \mathbf{c}_{l}^{+} \\
\mathbf{X}_{l+1} \mathbf{c}_{l+1}^{-}
\end{array}\right]+\left[\begin{array}{c}
\mathbf{f}_{l}^{1} \\
\mathbf{f}_{l}^{2}
\end{array}\right] .
$$

In order to compute the intermediary coefficients a recursion from bottom to top is employed. From the first equation of (57) we can compute

$$
\mathbf{X}_{l} \mathbf{c}_{l}^{+}=\left(\mathbf{S}_{l}^{\prime 11}\right)^{-1}\left(\mathbf{c}_{l+1}^{+}-\mathbf{S}_{l}^{\prime 12} \mathbf{X}_{l+1} \mathbf{c}_{l+1}^{-}-\mathbf{f}_{l}^{1}\right),
$$

which may be used in the second equation of (57) to compute $\mathbf{c}_{l}^{-}$

$$
\begin{aligned}
\mathbf{c}_{l}^{-}= & \mathbf{S}_{l}^{\prime 21}\left(\mathbf{S}_{l}^{\prime 11}\right)^{-1} \mathbf{c}_{l+1}^{+}+\left(-\mathbf{S}_{l}^{\prime 21}\left(\mathbf{S}_{l}^{\prime 11}\right)^{-1} \mathbf{S}_{l}^{\prime 12}+\mathbf{S}_{l}^{\prime 22}\right) \mathbf{X}_{l+1} \mathbf{c}_{l+1}^{-} \\
& -\mathbf{S}_{l}^{\prime 21}\left(\mathbf{S}_{l}^{\prime 11}\right)^{-1} \mathbf{f}_{l}^{1}+\mathbf{f}_{l}^{2} .
\end{aligned}
$$




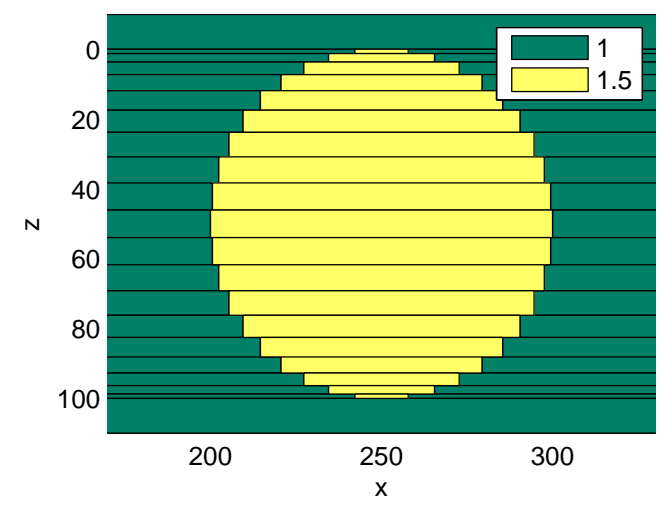

Fig. 3. Sliced geometry of a cylinder. Different colors correspond to different refraction indices.

Finally, the $\mathbf{c}_{l}^{+}$is computed from the first equation of (48),

$$
\mathbf{c}_{l}^{+}=\overline{\mathbf{S}}_{l-1}^{11} \mathbf{c}_{1}^{+}+\overline{\mathbf{S}}_{l-1}^{12} \mathbf{c}_{l}^{-}+\overline{\mathbf{f}}_{l-1}^{1} .
$$

As before, due to the absence of an incoming field in the superstrate, $\mathbf{c}_{1}^{+}=\mathbf{0}$, and $(60)$ becomes

$$
\mathbf{c}_{l}^{+}=\overline{\mathbf{S}}_{l-1}^{12} \mathbf{c}_{l}^{-}+\overline{\mathbf{f}}_{l-1}^{1} .
$$

Note that also in the computation of intermediary coefficients, the blocks $\overline{\mathbf{S}}_{l-1}^{11}$ and $\overline{\mathbf{S}}_{l-1}^{21}$ are not required. Thus, the computation of intermediary coefficients requires an additional sweep through the slices using recursive relations (59) and (61). The procedure is formally described by the diagram

$$
\mathbf{c}_{l+1} \stackrel{\left(\mathbf{S}_{l}, \mathbf{f}_{l}\right),\left(\overline{\mathbf{S}}_{l-1}, \overline{\bar{l}}_{l-1}\right)}{\longrightarrow} \mathbf{c}_{l} .
$$

The recursion is initialized at $l=M$, with $\mathbf{c}_{M}^{-}=\mathbf{0}, \mathbf{c}_{M}^{+}=\overline{\mathbf{f}}_{M-1}^{1}$.

\section{Numerical results}

We consider the problem of scattering of a plane wave from a dielectric cylinder. An important argument for this choice is the fact that a semi-analytical solution can be found. The solution of the cylinder problem is obtained by writing the Maxwell equations in cylindrical coordinates, expanding the fields inside and outside the cylinder in terms of Bessel functions and finally matching the fields at the cylinder's interface [18]. 

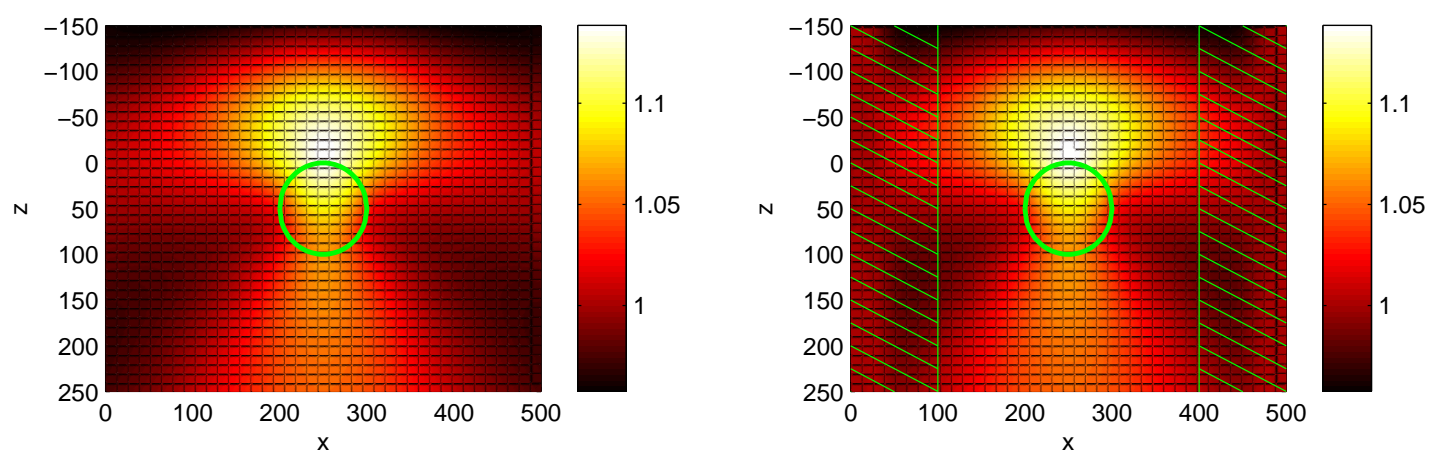

Fig. 4. Absolute values of the electric field: exact solution (left) and solution computed with aFMM-CFF (right). Hatched areas indicate PMLs.

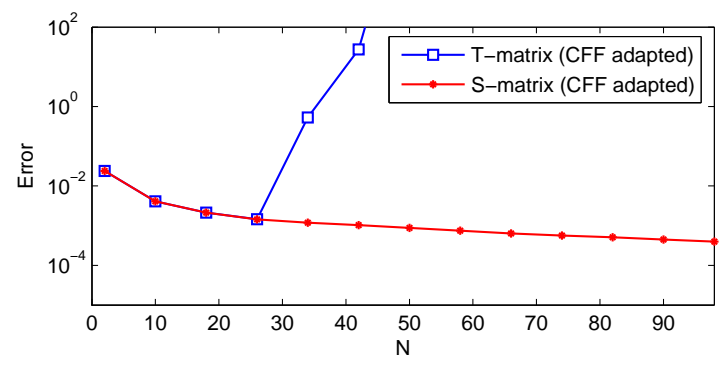

Fig. 5. Convergence of the aFMM-CFF with the adapted S-matrix and Tmatrix approaches for a cylinder with radius $\rho=50 \mathrm{~nm}$ approximated by $M=79$ slices.

In order to solve the same problem with aFMM-CFF, we first need to approximate the geometry by multiple rectangular slices. For a cylinder this may be done by imposing a fixed arc-length between two adjacent slices. This will ensure an adaptive, slope-dependent staircasing of the profile. Figure 3 shows such a profile for a cylinder with radius $\rho=50$ nm obtained using $M=19$ slices. The incident plane wave has a wavelength $\lambda=628.3 \mathrm{~nm}$, travels downwards in the plane perpendicular to the cylinder and is TM-polarized. The semianalytical solution and the corresponding aFMM-CFF solution of this problem are shown in Figure 4. The PMLs are placed in the stripes $x \in[0,100]$ and $x \in[400,500]$ and implemented using the coordinate transformation (3) with the parameters $\sigma_{0}=20, p=1$. As expected, in the region between the PMLs the numerical solution is close to the reference solution.

We now turn our attention to the issue of stability. We use the cylinder problem to com- 
pare the performance of a straightforward T-matrix algorithm adapted for non-homogeneous linear systems and the modified S-matrix algorithm presented in Section 3. Figure 5 shows the convergence of aFMM-CFF combined with these approaches for a cylinder with radius $\rho=50 \mathrm{~nm}$ approximated by $M=79$ slices. The error is defined as

$$
\mathcal{E}=\left\|h_{y}^{t, N}(x, z)-h_{y}^{t, r e f}(x, z)\right\|_{2}, \text { on } \Omega_{s},
$$

where $h_{y}^{t, N}$ is the numerical solution obtained with aFMM-CFF for $2 N+1$ harmonics, $h_{y}^{t, r e f}$ is the semi-analytical solution for the cylinder problem, and $\Omega_{s}=[200,300] \times[0,100]$ is the smallest rectangular domain enclosing the cylinder.

\begin{tabular}{llll}
\hline & $\rho=25 \mathrm{~nm}$ & $\rho=50 \mathrm{~nm}$ & $\rho=100 \mathrm{~nm}$ \\
\hline$N=10$, S-matrix & $1.0261 \mathrm{e}+00$ & $1.0600 \mathrm{e}+00$ & $9.2628 \mathrm{e}-01$ \\
$N=10$, T-matrix & $1.0261 \mathrm{e}+00$ & $1.0600 \mathrm{e}+00$ & $9.2628 \mathrm{e}-01$ \\
\hline$N=20$, S-matrix & $1.0264 \mathrm{e}+00$ & $1.0602 \mathrm{e}+00$ & $9.2614 \mathrm{e}-01$ \\
$N=20$, T-matrix & $1.0264 \mathrm{e}+00$ & $1.0602 \mathrm{e}+00$ & $1.2620 \mathrm{e}+00$ \\
\hline$N=40$, S-matrix & $1.0265 \mathrm{e}+00$ & $1.0602 \mathrm{e}+00$ & $9.2629 \mathrm{e}-01$ \\
$N=40$, T-matrix & $1.0265 \mathrm{e}+00$ & $1.4295 \mathrm{e}+00$ & $6.5550 \mathrm{e}+19$ \\
\hline$N=80$, S-matrix & $1.0266 \mathrm{e}+00$ & $1.0602 \mathrm{e}+00$ & $9.2636 \mathrm{e}-01$ \\
$N=80$, T-matrix & $1.0167 \mathrm{e}+00$ & $3.6577 \mathrm{e}+19$ & $6.1624 e+61$ \\
\hline Reference & $1.0266 \mathrm{e}+00$ & $1.0602 \mathrm{e}+00$ & $9.2637 \mathrm{e}-01$ \\
\hline
\end{tabular}

Table 1. Magnitudes of the magnetic field in a fixed point above the cylinder computed with the T-matrix and S-matrix algorithms adapted for CFF for increasing radius $\rho$ and truncation order $N$.

For a low truncation number $N$, both algorithms give similar results. At $N>30$ the Tmatrix algorithm becomes unstable while the S-matrix algorithm yields accurate results for a larger number of harmonics. The truncation number for which the algorithm breaks down depends on the slice thickness, as demonstrated in Table 1. It lists the magnitudes of the magnetic field in a point $(250,-100)$ computed with the two approaches. The radius of the cylinder $\rho$ and the truncation order $N$ are varying. From the matrix $\mathbf{X}_{l}=e^{-k_{0} \mathbf{Q}_{l}\left(h_{l+1}-h_{l}\right)}$ it is visible that increasing the radius of the cylinder while keeping a fixed number of slices (thus increasing slice thickness) or increasing the number of harmonics will lead to smaller entries in $\mathbf{X}_{l}$ and respectively larger entries in its inverse, generating significant round-off errors. This explains the instabilities exhibited by the extended T-matrix approach for larger values of $N$ and $\rho$. On the other hand, the modified S-matrix algorithm is stable and gives correct 
results for all $N$ and $\rho$. The offset of the computed solution with respect to the reference solution visible in Table 1 and the plateau on Figure 5 (error remains constant for $N>50$ ) are due to staircasing. This effect is well known for the FMM [19] and can be reduced by a normal vector field approach [20].

\section{Conclusion}

The aFMM-CFF has been generalized for use with arbitrary profiles. The cases of planar (TE, TM) and conical incidence have been briefly presented. The existing S-matrix algorithm has been adapted for use with the new type of recursive linear systems. For this purpose, a set of recursive relations has been derived which give a stable algorithm for the computation of reflection and transmission coefficients. Moreover, a backward recursion may be employed to determine the intermediary coefficients if needed. The stability of the developed method has been qualified by comparison against a semi-analytical result obtained for the problem of scattering of a plane wave from a dielectric cylinder

\section{Acknowledgments}

Authors would like to thank ASML B.V. for funding this project. We are also grateful to Teis Coenen and Ronald Rook for assistance in obtaining the reference solution.

\section{References}

1. M. G. Moharam, E. B. Grann, D. A. Pommet, and T. K. Gaylord, "Formulation for stable and efficient implementation of the rigorous coupled-wave analysis of binary gratings," J. Opt. Soc. Am. A 12, 1068-1076 (1995).

2. E. Popov and M. Nevière, "Grating theory: new equations in Fourier space leading to fast converging results for TM polarization," J. Opt. Soc. Am. A 17, 1773-1784 (2000).

3. M. Pisarenco, J. Maubach, I. Setija, and R. Mattheij, "Aperiodic Fourier modal method in contrast-field formulation for simulation of scattering from finite structures," J. Opt. Soc. Am. A 27, 2423-2431 (2010).

4. L. Li, "Multilayer modal method for diffraction gratings of arbitrary profile, depth, and permittivity," J. Opt. Soc. Am. A 10, 2581-2591 (1993).

5. D. M. Pai and K. A. Awada, "Analysis of dielectric gratings of arbitrary profiles and thicknesses," J. Opt. Soc. Am. A 8, 755-762 (1991).

6. L. F. DeSandre and J. M. Elson, "Extinction-theorem analysis of diffraction anomalies in overcoated gratings," J. Opt. Soc. Am. A 8, 763-777 (1991).

7. N. Chateau and J.-P. Hugonin, "Algorithm for the rigorous coupled-wave analysis of grating diffraction,” J. Opt. Soc. Am. A 11, 1321-1331 (1994). 
8. D. Y. K. Ko and J. R. Sambles, "Scattering matrix method for propagation of radiation in stratified media: attenuated total reflection studies of liquid crystals," J. Opt. Soc. Am. A 5, 1863-1866 (1988).

9. M. G. Moharam, D. A. Pommet, E. B. Grann, and T. K. Gaylord, "Stable implementation of the rigorous coupled-wave analysis for surface-relief gratings: enhanced transmittance matrix approach," J. Opt. Soc. Am. A 12, 1077-1086 (1995).

10. E. L. Tan, "Note on formulation of the enhanced scattering- (transmittance-) matrix approach," J. Opt. Soc. Am. A 19, 1157-1161 (2002).

11. M. van Kraaij, "Forward diffraction modelling: analysis and application to grating reconstruction," Ph.D. thesis (2011).

12. U. M. Ascher, R. M. M. Mattheij, and R. D. Russell, Numerical Solution of Boundary Value Problems for Ordinary Differential Equations (Cambridge University Press, 1995).

13. L. Li, "Formulation and comparison of two recursive matrix algorithms for modeling layered diffraction gratings," J. Opt. Soc. Am. A 13, 1024-1035 (1996).

14. H. Kim, I.-M. Lee, and B. Lee, "Extended scattering-matrix method for efficient full parallel implementation of rigorous coupled-wave analysis," J. Opt. Soc. Am. A 24, 2313-2327 (2007).

15. J. Berenger, "A perfectly matched layer for the absorption of electromagnetic waves," Journal of Computational Physics 114, 185-200 (1994).

16. W. C. Chew, J. M. Jin, and E. Michielssen, "Complex Coordinate Stretching as a Generalized Absorbing Boundary Condition," Microwave and Optical Technology Letters 15, 363-369 (1997).

17. L. Li, "Use of Fourier series in the analysis of discontinuous periodic structures," J. Opt. Soc. Am. A 13, 1870-1876 (1996).

18. J. R. Wait, "Scattering of a Plane Wave from a Circular Dielectric Cylinder at Oblique Incidence," Canadian Journal of Physics 33, 189-195 (1955).

19. E. Popov, M. Nevière, B. Gralak, and G. Tayeb, "Staircase approximation validity for arbitrary-shaped gratings," J. Opt. Soc. Am. A 19, 33-42 (2002).

20. T. Schuster, J. Ruoff, N. Kerwien, S. Rafler, and W. Osten, "Normal vector method for convergence improvement using the RCWA for crossed gratings," J. Opt. Soc. Am. A 24, 2880-2890 (2007). 
PREVIOUS PUBLICATIONS IN THIS SERIES:

\begin{tabular}{|c|c|c|c|}
\hline Number & Author(s) & Title & Month \\
\hline III-35 & $\begin{array}{l}\text { E.J. Brambley } \\
\text { M. Darau } \\
\text { S.W. Rienstra }\end{array}$ & $\begin{array}{l}\text { The critical layer in } \\
\text { sheared flow }\end{array}$ & May 'II \\
\hline II-36 & $\begin{array}{l}\text { M. Oppeneer } \\
\text { W.M.J. Lazeroms } \\
\text { S.W. Rienstra } \\
\text { R.M.M. Mattheij } \\
\text { P. Sijtsma }\end{array}$ & $\begin{array}{l}\text { Acoustic modes in a duct } \\
\text { with slowly varying } \\
\text { impedance and non- } \\
\text { uniform mean flow and } \\
\text { temperature }\end{array}$ & May 'II \\
\hline II-37 & $\begin{array}{l}\text { M.E. Hochstenbach } \\
\text { N. Mcninch } \\
\text { L. Reichel }\end{array}$ & $\begin{array}{l}\text { Discrete ill-posed least- } \\
\text { squares problems with a } \\
\text { solution norm constraint }\end{array}$ & June 'II \\
\hline II -38 & $\begin{array}{l}\text { T. Fatima } \\
\text { A. Muntean } \\
\text { M. Ptashnyk }\end{array}$ & $\begin{array}{l}\text { Unfolding-based corrector } \\
\text { estimates for a reaction- } \\
\text { diffusion system } \\
\text { predicting concrete } \\
\text { corrosion }\end{array}$ & June 'II \\
\hline II-39 & $\begin{array}{l}\text { M. Pisarenco } \\
\text { J.M.L. Maubach } \\
\text { I. Setija } \\
\text { R.M.M. Mattheij }\end{array}$ & $\begin{array}{l}\text { A modified S-matrix } \\
\text { algorithm for the aperiodic } \\
\text { Fourier modal method in } \\
\text { contrast-field formulation }\end{array}$ & June 'II \\
\hline
\end{tabular}

